\title{
Network Traffic Analysis and QoE evaluation for Video Progressive Download service: Netflix
}

\author{
Francisco Lozano, Mari-Carmen Aguayo-Torres, Gerardo Gómez, Carlos \\ Cárdenas, and Juana Baños \\ Universidad de Málaga, Departamento de Ingeniería de Comunicaciones, Campus de \\ Teatinos, 29071, Málaga, Spain \\ Tel.: +34-952-134168 \\ \{flg, aguayo\}@ic.uma.es \\ http://www.ic.uma.es
}

\begin{abstract}
Over the Top video streaming services has grown very rapidly in recent years, with the emerge of diverse online video stores. One of the popular over the top services is Netflix. The significant increase of user data consumption by this type of services affects the performance of communications networks, and operators need methods to estimate how well the network behaves. In this paper a network traffic analysis of Netflix is presented. The traffic study has been performed with diverse devices and access technologies. A model for quality of experience (QoE) evaluation, based on application performance metrics, has been applied to estimate Mean Opinion Score (MOS) by end users.
\end{abstract}

Keywords: Traffic Pattern, Quality of Experience, Mean Opinion Score, Netflix ${ }^{\text {TM }}$

\section{Introduction}

Mobile video traffic represents today $50 \%$ of all data traffic and, according to forecasts for 2017 , video traffic on mobile devices will account for $66 \%$ of the total traffic for these terminals [1]. Netflix is a providing services platform mainly via streaming video on demand. It began in the United States and is slowly expanding its services to other countries in America and Europe. Online distribution began in 1999 and in 2015 has more than 50 million subscribers. This outstanding traffic demand growth represents a serious challenge for network operators, who should engineer their wireless networks architecture to handle the huge volume of traffic in efficient ways while providing the highest Quality of Experience (QoE) to end users. Netflix platform has adapted to computers, TVs and mobile devices, allowing users access to digital content from smartphones and tablets via wireless networks. In terms of communication protocols, Netflix uses Hypertext Transfer Protocol (HTTP) to transfer video instead of Real-time Transport Protocol (RTP) over User Datagram Protocol (UDP), as could be expected for a real time service. 
Perceived user quality results from a mix of performance indicators that are different from one application to another. In the case of video applications, any stop during the rendering of the image when the user is watching a video is identified as lack of quality.

A traditional way to evaluate the quality perceived by users is by performing a user survey under various network conditions. The ratings are averaged over a large sample of users in order to obtain a single parameter known as "Mean Opinion Score" (MOS) [3]. This approach has several drawbacks such as the difficulty in ensuring repeatability of the network conditions and the cost of the survey itself. Hence, it would be more appropriate to automate the measurement procedure so that it could be performed without human intervention. To this end, MOS models have to be developed in order to substitute direct user opinion on services' performance. Once models that correlate specific measurable parameters to user perceived quality are available, network operators can estimate the perceived user quality from the measurement of those parameters.

The rest of this paper is organized as follows. Section 2 presents an analysis of the network traffic pattern of Netflix. In Section 3, a QoE evaluation model for the type of traffic of Netflix is described. Finally, some concluding remarks are given in the last section.

\section{Network traffic patterns}

Netflix is based on adaptive video streaming over HTTP [5]. The main difference between this type of service and real-time video streaming is that the video content has been previously stored in multimedia server. The service provider settles copies of the same movie classified by different qualities. Each movie file is also segmented into fragments.

Basic functionality description of this mechanism is illustrated in Figure 1. At the beginning the player client requests a manifest, which includes the information of the coding rates available of the movie selected. Then, the client requests an specific fragment of video file from server. The adaptive mechanism is based in changing the quality of the movie fragment as function of bandwidth available or congestion detection.

The adaptive mechanism is based on changing the next movie fragment quality, as function of the bandwidth available or network congestion. For example in figure 1 at time $t=2$, the client request at best available encoding rate fragment for the already video playing, then a congestion episode occurs so the client requests a fragment with lower encoding rate avoiding stalls during the video playback. When the conditions are acceptable the client will request fragments at higher available quality.

The profile of the traffic generation mechanism in an ideal scenario shows two main phases, Initial data burst and Control algorithm. Figure 2 shows both phases, being blue zone the Initial Burst and the red one the Control Algorithm phase. 


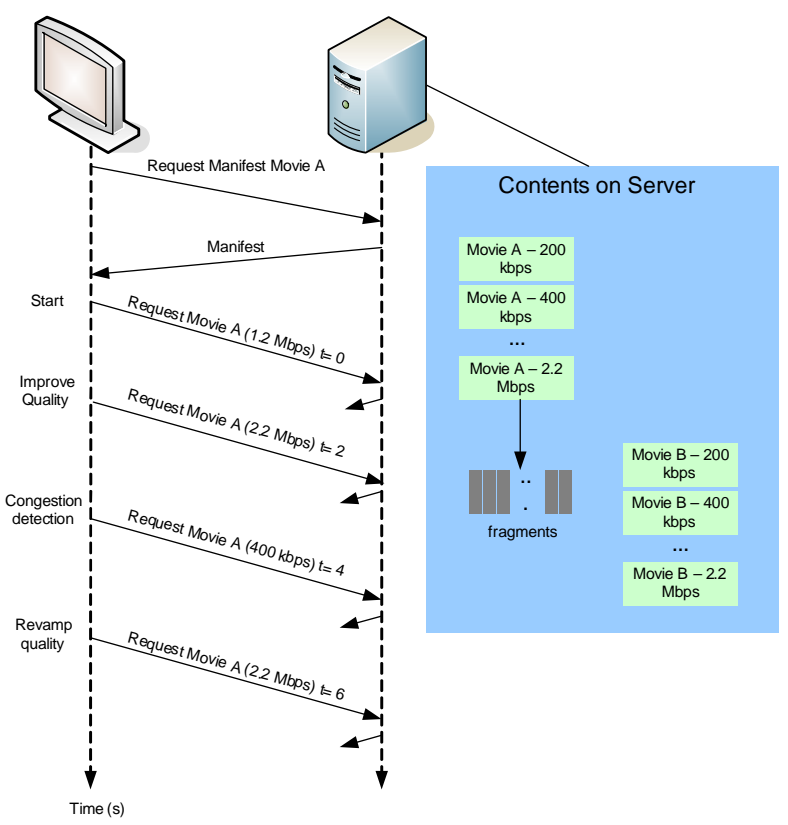

Fig. 1. Adaptive HTTP video streaming

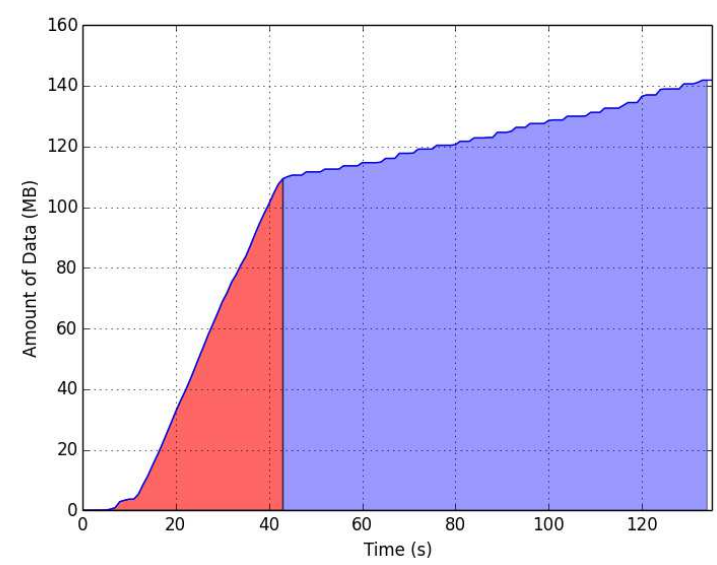

Fig. 2. Phases of video download 


\subsection{Initial Data Burst}

Corresponds to data transmission before video playback at the client. This state is also known as buffering time, which is the time to download a certain amount of data to play the video with the best quality available, avoiding stalls at the beginning. The amount of data downloaded during the buffering time matches with 300 seconds of real video playback time, the input parameter is the video rate encoding $\left(V_{r}\right)$ of the selected video, as depicted in equation 1.

$$
\text { Total Amount of Data } \text { Duffering }=300 V_{r}
$$

\subsection{Control algorithm}

Control Algorithm is characterized by low throughput generated from server and a typical traffic profile with ON-OFF cycles of transmission, throwing a curve with steps as shown in blue zone of figure 2 . The goal of this phase is to ensure progressive download $25 \%$ faster than video file playback on the client player. Hence, in ideal conditions there are not any stall during the video playback at client viewer.

\subsection{Experimental Setup}

A test plan was carried out to model the Netflix traffic pattern. Table 1 presents multiple devices to analyze radio access technologies under test. Due to availability, we just performed records over these technologies and devices with Netflix access.

Table 1. Platforms under test

\begin{tabular}{ccc}
\hline Device & WLAN (802.11n) Cellular (LTE) \\
\hline Laptop (Windows) & $\mathrm{X}$ & \\
Smartphone (Android) & $\mathrm{X}$ & $\mathrm{X}$ \\
Tablet (Android) & $\mathrm{X}$ & \\
\hline
\end{tabular}

Two type of movies was selected to simulate different group of source. The classification is based on the characteristics of the movie content by the image transitions as presented in table 2. The capture of video traffic was performed during 200 seconds with several iterations to ensure a reproducible measurement.

\subsection{Comparison}

Under WLAN 802.11n coverage we performed records for different devices and establish a comparison between them. First result we obtained is the traffic pattern with two phases, previously described, but with significant differences 
Table 2. Video traces

\begin{tabular}{clc}
\hline Video Trace Description & Acquisition Time \\
\hline$\# 1$ & $\begin{array}{l}\text { Slower image transitions } \\
\# 2\end{array}$ & $\begin{array}{l}\text { Colorful and faster image } \\
\text { transitions }\end{array}$ \\
\hline
\end{tabular}

between client players. Figure 3 displays the amount of data downloaded versus time. The initial data burst corresponds to a lineal function with diverse slope for each device tested. For tests in PC under WLAN connection, the initial data burst downloads more amount of data but over more time, on the opposite side, smartphones running Android OS with native Netflix client, adopts a criteria with less data downloaded during this phase. The Control algorithm also shows a distinctive behavior, while PC and Tablet download more periodically but less amount of data, Smartphones download more data per chunk, but with less periodicity.

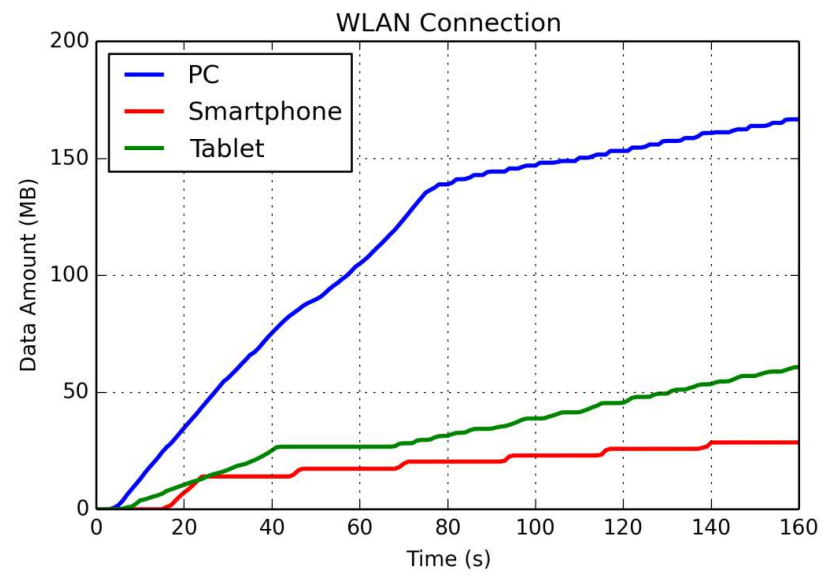

Fig. 3. Comparison between devices

In-depth analyze of the Control algorithm exhibits a different pattern usage in time and data downloaded. The experiments describe a reproducible CDF in both magnitudes. Figure 4 illustrates the out of line operation on Smarthphone devices, in terms of elapsed time between every chunk of data, with a mean value of 24 seconds. For PC and Tablet the response are quite similar nearby 4 seconds. 


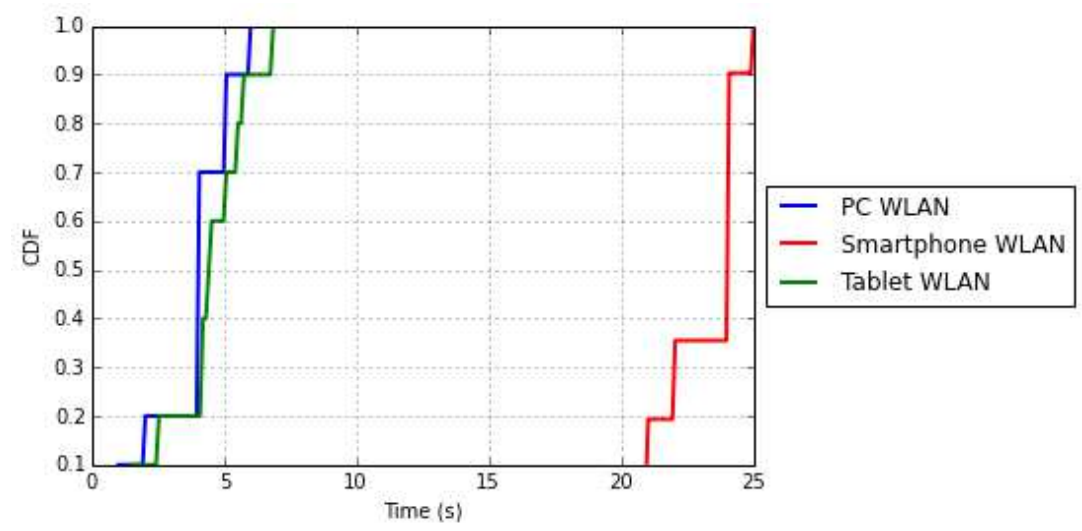

Fig. 4. Time CDF by device

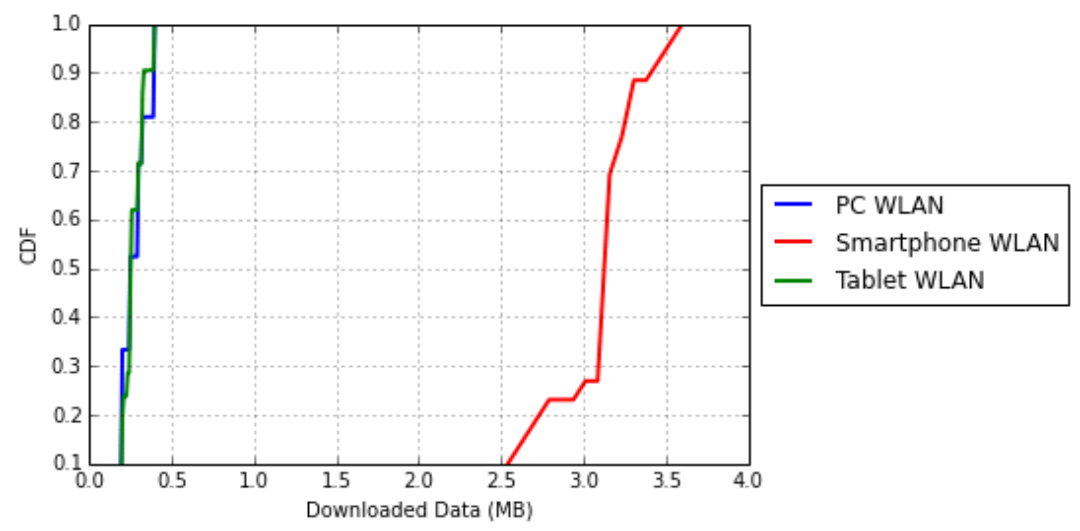

Fig. 5. Data CDF by device

The amount of data received per chunk, under the Control algorithm, also shows diverse operation over the devices tested. Smarthphone presents a mean value of $4 \mathrm{MB}$ per chunk while $\mathrm{PC}$ and Tablet have less than $0.5 \mathrm{MB}$, as is depicted by the CDF of the received data in figure 5 .

Based on our empirical study we propose a Netflix traffic model, classified by two phases, initial data burst and control algorithm. Table 3 summarize 
the parameters of the model for Netflix. A detailed pseudo-code of the control algorithm is also presented.

Table 3. Proposed Netflix traffic model

\begin{tabular}{ccc}
\hline Phase & Initial Data Burst & Control algorithm \\
\hline Amount of Data to send (bits) & $300 V_{r}$ & Video length - $300 V_{r}$ \\
TCP Packet size & MSS & MSS \\
Source speed & Full Buffer TCP & Client platform function (code below) \\
\hline
\end{tabular}

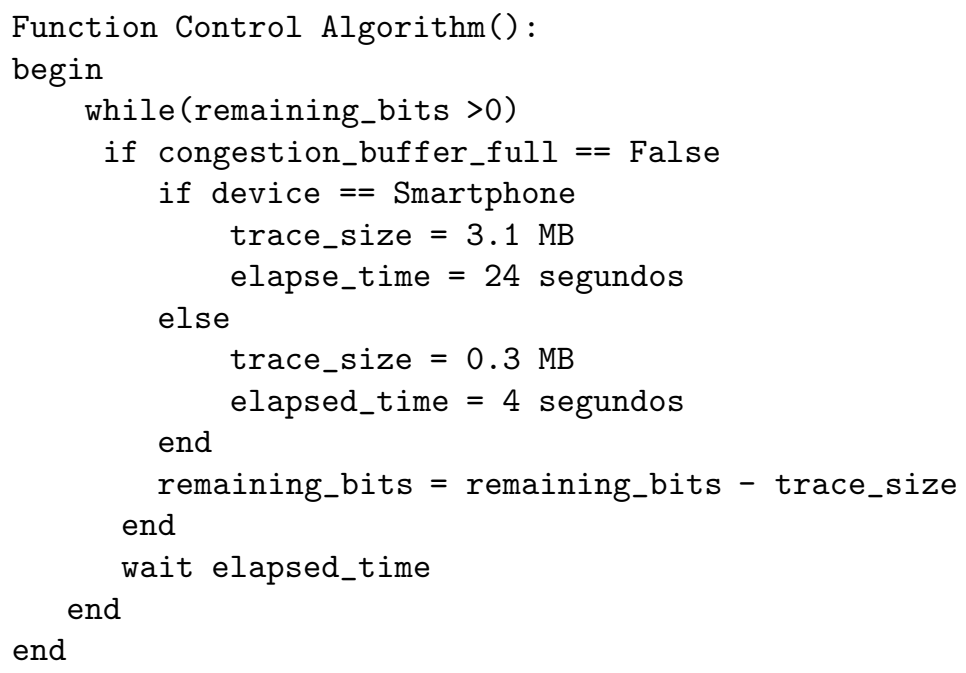

\section{QoE Evaluation}

We have employed the QoE evaluation for multimedia services based on the ITU-T Recommendation P.800 [3]. This opinion scale, the most frequently used for multimedia services, allocates qualitative values from Bad to Excellent by mapping the quantitative MOS as depicted in table 4. A minimum value of 3 has to be obtained to establish quality as Fair, being 5 the maximum score and 1 the lowest mark.

In adaptive HTTP video streaming service the following APMs are required by the model in [4][2]:

- Initial buffering time $\left(T_{\text {init }}\right)$ : period between the starting time of loading a video and the starting time of playing it.

- Rebuffering frequency $\left(f_{\text {rebuf }}\right)$ : frequency of interruption events during the playback.

- Mean rebuffering time $\left(T_{\text {rebuf }}\right)$ : average duration of a rebuffering event. 
Table 4. ITU-T Recommendation P.800 MOS scale [3]

\begin{tabular}{cc}
\hline Quality & MOS \\
\hline Excellent & 5 \\
Good & 4 \\
Fair & 3 \\
Poor & 2 \\
Bad & 1 \\
\hline
\end{tabular}

Finally, the MOS is computed as a linear model as [2][4]:

$$
M O S=4.23-0.0672 L_{T_{i}}-0.742 L_{f_{r}}-0.106 L_{T_{r}}
$$

where $L_{T_{i}}, L_{f_{r}}$ and $L_{T_{r}}$ are quantized values of the respective levels $T_{\text {init }}, f_{\text {rebuf }}$ and $T_{\text {rebuf }}$, following Table 5 .

Table 5. APMs quantification levels

\begin{tabular}{|c|c|c|c|}
\hline & $T_{i n i t}$ & $f_{\text {rebuf }}$ & $T_{\text {rebuf }} L$ \\
\hline Low & $0-1 \mathrm{~s}$ & $0-0.02$ & $0-5 \mathrm{~s}$ \\
\hline Medium & $1-5 \mathrm{~s}$ & $0.02-0.15$ & $5-10 \mathrm{~s} 2$ \\
\hline High & $>5 \mathrm{~s}$ & $>0.15$ & $>10 \mathrm{~s} \quad 3$ \\
\hline
\end{tabular}

Note that the parameter which affects worst to proposed mean opinion score model, is related to the rebuffering frequency. In terms of the user perception this parameter measure the number of stalls may occur during the video playback.

\section{Conclusion}

This work has presented main characteristics of traffic generation mechanism of Netflix with two clearly different parts, an Initial data burst and a Control algorithm. Our research conducts to describe the transmission of amount of data which corresponds to 300 seconds of real video playback, depending of the video encoding employed.

The QoE evaluation has been performed with a proposed model, based on application performance metrics by the client side. The goal of the model is to obtain a mean opinion score with just 3 parameters.

Acknowledgments This work has been partly supported by the Spanish Government and FEDER under grant TEC2013-44442-P.

The authors would like to thank Noelia Guerra Melgares for her collaboration and technical support during the measurement process. 


\section{References}

1. Cisco Systems Inc.: Cisco visual networking index: Forecast and methodology, 20132018. http://www.cisco.com (2013)

2. Gómez, G., Hortigüela, L., Pérez, Q., Lorca, J., García, R., Aguayo-Torres, M.C.: Youtube qoe evaluation tool for android wireless terminals. EURASIP Journal on Wireless Communications and Networking 2014(164) (2014)

3. ITU-T: Recommendation P.800. Methods for subjective determination of transmission quality (August 1996)

4. Mok, R., Chan, E., Chang, R.: Measuring the quality of experience of http video streaming. In: Integrated Network Management (IM), 2011 IFIP/IEEE International Symposium on. pp. 485-492 (May 2011)

5. Rao, A., Legout, A., Yeon-sup, L., Towsley, C., Barakat, C., D.W.: Network characteristics of video streaming traffic. In: Proceedings of the Seventh Conference on Emerging Networking Experiments and Technologies (2011) 